\title{
Association between premature ovarian insufficiency and gut microbiota
}

Jiaman $\mathrm{Wu}^{1 \dagger}$, Yuanyuan Zhuo ${ }^{2 \dagger}$, Yulei Liu', Yan Chen ${ }^{1}$, Yan Ning ${ }^{1 *}$ and Jilong Yao ${ }^{{ }^{*}}$

\begin{abstract}
Background: Premature ovarian insufficiency (POI) is characterized by impairment of ovarian function on a continuum before the age of 40 years. $\mathrm{POI}$ is affected by multiple factors. Considering new insights from recent gut microbiome studies, this study aimed to investigate the relationship between gut microbial community structure and POI.

Methods: Subjects were recruited at the Shenzhen Maternity \& Child Healthcare Hospital. Fecal microbial community profiles of healthy women $(n=18)$, women with POI $(n=35)$ were analyzed using 165 rRNA gene sequencing based on Illumina NovaSeq platform.

Results: Compared to the controls, the serum levels of FSH, LH, T and FSH/LH ratio significantly increased in women with POI, whereas E2 and AMH decreased significantly. Higher weighted UniFrac value was observed in POI women compared with healthy women. Phylum Firmicutes, genera Bulleidia and Faecalibacterium were more abundant in healthy women, while phylum Bacteroidetes, genera Butyricimonas, Dorea, Lachnobacterium and Sutterella enriched significantly in women with POI. Moreover, these alterations of the gut microbiome in women with $\mathrm{POI}$ were closely related to FSH, LH, E2, AMH level and FSH/LH ratio.
\end{abstract}

Conclusions: Women with POI had altered microbial profiles in their gut microbiome, which were associated with serum hormones levels. These results will shed a new light on the pathogenesis and treatment for POI.

Keywords: Premature ovarian insufficiency, Gut microbiota, Sex hormones, 165 rRNA sequencing

\section{Background}

Premature ovarian insufficiency (POI) is an ovarian insufficiency syndrome before the age of 40 years affecting approximately $1-2 \%$ women $[26,12]$. It is characterized by a continuous decline in ovarian function, and resulting in an earlier cessation of menstruation than normal [12]. Women with POI are faced with increased risk of low chance of natural conception [4, 38], urogenital atrophy [34], decrease in bone mineral density [3],

\footnotetext{
*Correspondence: ningjudy@163.com; yaojilong369@163.com

†Jiaman Wu and Yuanyuan Zhuo are co-first authors and contributed equally to this work.

'Affiliated Shenzhen Maternity\&Child Healthcare Hospital, Southern Medical University, No. 3012, Fuqiang Road, Futian District, Shenzhen City 518000, Guangdong Province, China

Full list of author information is available at the end of the article
}

autoimmune and thyroid disease risk [11], cognitive dysfunction [18], shortened life expectancy [30], and cardiovascular disease [44, 15]. POI is a multifactorial disease [33]. Spontaneous POI is associated with genetic defects, autoimmunue diseases, enzyme deficiency and environmental factors, and iatrogenic POI occurs mainly due to surgical intervention, chemotherapy and radiotherapy $[18,33]$.

There is ample evidence indicating that ovary is damaged by autoimmunity through alteration of T-cell subsets, T-cell-mediated injury, increasing of autoantibodyproducing B-cells, decreasing of effector suppressor/cytotoxic lymphocytes, and decreasing of natural killer cells [11]. It supports that antoimmune etiology exists in POI based on the presence of lymphocytic oophoritis, association with autoimmune disorders, and autoantibodies [11, 21]. A number of studies have demonstrated that gut microbiome

(c) The Author(s). 2021 Open Access This article is licensed under a Creative Commons Attribution 4.0 International License, which permits use, sharing, adaptation, distribution and reproduction in any medium or format, as long as you give appropriate credit to the original author(s) and the source, provide a link to the Creative Commons licence, and indicate if changes were made. The images or other third party material in this article are included in the article's Creative Commons licence, unless indicated otherwise in a credit line to the material. If material is not included in the article's Creative Commons licence and your intended use is not permitted by statutory regulation or exceeds the permitted use, you will need to obtain permission directly from the copyright holder. To view a copy of this licence, visit http://creativecommons.org/licenses/by/4.0/ The Creative Commons Public Domain Dedication waiver (http://creativecommons.org/publicdomain/zero/1.0/) applies to the data made available in this article, unless otherwise stated in a credit line to the data. 
play a key role in the autoimmune process [45]. Such as the peptides generated by gut microbiota could induce immune cells to become autoreactive and crossactivated [45]. Moreover, the dysbiosis of gut microbiome not only can affect the activation of B lymphocytes and the production of autoantibodies, but also induce the aberrant activation of innate immune cells, which leads to the upregulation of proinflammatory cytokines [45]. In addition, increasing evidences have strongly suggested that gut microbiome play an important role in POIassociated symptoms, including autoimmune dysfunction $[39,8]$, bone health $[20,29]$, cognitive and neurological health [28, 14]. Gut microbiota and its metabolites also have the ability to regulate inflammation pathway activation, brain-gut peptide secretion and the destruction of islet $\beta$-cell $[46,22]$. All these studies indicate a relationship may exist between the gut microbiome and POI.

In order to study the community profile of gut microbiome in women with POI, and how the changes of gut microbiota correlated with the sex hormones, 35 women with POI and 18 healthy women were recruited in this study. Sequencing of the V3-V4 regions of $16 \mathrm{~S}$ rRNA gene in fecal samples was performed to reveal the substantial differences of gut microbiota between the POI subjects and controls.

\section{Methods}

\section{Study cohort}

A total of 35 women with spontaneous POI and 18 healthy women, aged 24 to 40 years, were recruited at the Shenzhen Maternity\&Child Healthcare Hospital from August 2019 to September 2019. Spontaneous POI was diagnosed and assessed according to the previously reported [17], primary or secondary amenorrhea for at least 4 months before 40 years, and with at least two instances of serum follicle-stimulating hormone (FSH) levels exceeded $40 \mathrm{IU} / \mathrm{L}$ with an interval of 4-6 weeks. All the control women had normal ovarian function, without history of menstrual dysfunction and infertility, with regular menstruation and normal levels of FSH (<10 IU/L). Participants were excluded if with following situations: non-46-XX karyotype, POI with family history, pregnancy, tumor, chronic diarrhea, autoimmune diseases, use of antibiotics/medications with the preceding 3 months, pelvic surgery, gastrointestinal disease, active infections, body mass index $<18.5$ or $>23.9 \mathrm{~kg} / \mathrm{m}^{2}$, smoking and chemo/ radiotherapy treatment. The study protocol was approved by the ethics committee of Shenzhen Maternity\&Child Healthcare Hospital. Written informed consents were obtained from all participants prior to enrollment. And clinical characteristics were extracted from the health records.

\section{Sampling}

All participants were examined in the morning after $>8$ $\mathrm{h}$ fasting. Fecal samples were collected using empty stool collection tubes with an inbuilt sterile swab. Samples were stored at $-80 \mathrm{C}$ until further analysis.

\section{DNA extraction and sequencing}

DNA was extracted from fecal samples using QIAamp DNA stool mini kit (Qiagen, Germany) according to the manufacturer's instructions. PCR amplification was conducted using 338F forward primer 5'-ACTCCTACGG GAGGCAGCAG-3' and 806R reverse primer 5'-GGAC TACHVGGGTWTCTAAT-3' targeting the variable V3V4 regions of $16 \mathrm{~S}$ rRNA gene. All samples were pooled equally and sequenced on an Illumina NovaSeq 6000 machine with $2 \times 250$ flow cell. Raw sequencing data in this study were deposited into the NCBI's Sequence Read Archive database (SRA BioProject ID PRJNA615330).

\section{Sequencing data analysis}

Sequencing reads were assigned to each sample based on dual-index barcodes using custom Perl script. Reads were processed using the bioinformatics software package QIIME2 (version 2019.10). Firstly, the reads were imported to a QIIME2 artifact with command "qiime tool import". Then the reads were denoised with command "qiime data 2 denoise-paired" to exclude chimeric sequences and phiX sequences. Next, taxonomy was assigned with command "qiime2 feature-classifier classify-sklearn" against Greengenes (13_8 revision) database. Meanwhile, Shannon index and weighted UniFrac distance were generated with command "qiime phylogeny align-to-tree-mafft-fasttree" and "qiime diversity core-metrics-phylogenetic" at a sample depth of 1000. Principal coordinate analysis (PCoA) based on weighted UniFrac distances were also calculated.

\section{Statistical analysis}

Permutational multivariate analysis of variance (PERMANOVA) was performed on weighted UniFrac distance to investigate the differences of microbial community structure between POI group and healthy control group using package vegan (999 permutations) in $\mathrm{R}$ software.

Statistical calculations were performed using R software, and $P$ value $<0.05$ was considered significantly different. Normality test was conducted using Shapiro-Wilk test. Unpaired $t$-tests were used for comparisons of clinical characteristics, indices of diversity between the two groups. All these continuous data were expressed as mean value \pm standard deviation (SD). While Wilcoxon Rank Sum tests were used for taxa comparisons at the phylum and genus level. Partial correlation analysis was used to investigate the relationships between microbes and clinical characteristics in R packages "ggm" and "psych". 


\section{Results}

\section{Study subject characteristics}

Study subjects are all Han Chinese and live in Shenzhen City. Their characteristics were summarized in Table 1. All participants aged from 24 to 40 years old (average: 34.6 years old), accompanied with body mass index (BMI) ranging from 18.6 to $23.9 \mathrm{~kg} / \mathrm{m}^{2}$ (average: 21.2 ). Statistical analysis demonstrated that there were no differences at age, progesterone (P), prolactin (PRL) and glucose (GLU) between women with and without POI. In addition, women with POI had significantly higher levels of BMI, FSH, luteinizing hormone (LH), testosterone (T) and FSH/LH ratio, but significantly lower levels of estradiol (E2) and anti-Mullerian hormone (AMH), compared to healthy control women.

\section{Overall community structure of POI gut microbiome}

Sequencing was performed on the V3-V4 regions of $16 \mathrm{~S}$ rRNA to evaluate the community structure of gut microbiota in women with and without POI. In total, 3,163, 487 usable reads $(59,688 \pm 11,095$ reads per sample) were obtained from all 53 samples, and the mean and median sequence lengths were 411 and $410 \mathrm{bp}$ separately. The number of reads analyzed did not differ between POI and control samples $(60,943 \pm 11,393$ versus $57,247 \pm 10$, 326, $P=0.25)$, indicating comparable and adequate sequencing coverage.

To explore the dissimilarity of gut microbiota between the two groups, PCoA analysis was performed based on the weighted UniFrac distance. The results showed the subjects of the two groups did not separate (Fig. 1a) $(P=0.16$, PERMANOVA analysis with 999 permutations). Further, POI subjects exhibited a higher Shannon index without significant difference between the two

Table 1 Demographic and clinical characteristics of the two groups

\begin{tabular}{llll}
\hline Characteristic & POI $=\mathbf{3 5}$ & NG $=\mathbf{1 8}$ & P value \\
\hline Age (years) & $35.23 \pm 4.62$ & $33.5 \pm 4.05$ & 0.19 \\
BMI $\left(\mathrm{kg} / \mathrm{m}^{2}\right)$ & $21.5 \pm 1.35$ & $20.54 \pm 1.61$ & $0.02^{*}$ \\
FSH $(\mathrm{mll} / \mathrm{mL})$ & $45.60 \pm 28.77$ & $5.39 \pm 1.74$ & $<0.01^{* *}$ \\
$\mathrm{LH}(\mathrm{mll} / \mathrm{mL})$ & $15.79 \pm 9.02$ & $4.04 \pm 1.07$ & $<0.01^{* *}$ \\
E2 $(\mathrm{pg} / \mathrm{L})$ & $30.71 \pm 11.7$ & $54.56 \pm 9.0$ & $<0.01^{* *}$ \\
P $(\mathrm{nmol} / \mathrm{L})$ & $0.48 \pm 0.33$ & $0.35 \pm 0.14$ & 0.12 \\
T $(\mathrm{nmol} / \mathrm{L})$ & $0.43 \pm 0.22$ & $0.31 \pm 0.11$ & $0.03^{*}$ \\
PRL $(\mathrm{nmol} / \mathrm{L})$ & $14.37 \pm 7.12$ & $11.49 \pm 4.57$ & 0.13 \\
AMH $(\mathrm{ng} / \mathrm{mL})$ & $0.49 \pm 0.35$ & $3.94 \pm 2.04$ & $<0.01^{* *}$ \\
FSH/LH $(\mathrm{ratio})$ & $2.88 \pm 0.68$ & $1.32 \pm 0.18$ & $<0.01^{* *}$ \\
GLU $(\mathrm{nmol} / \mathrm{L})$ & $5.07 \pm 0.41$ & $4.99 \pm 0.32$ & 0.48
\end{tabular}

$B M I$ body mass index, FSH follicle-stimulating hormone, $L H$ luteinizing hormone, E2 estradiol, $P$ progesterone, $T$ testosterone, $P R L$ prolactin, $A M H$ antiMullerian hormone, GLU glucose

${ }^{*} P<0.05 ;{ }^{* *} P<0.01$ groups $(4.97 \pm 0.74$ versus $4.71 \pm 0.39, P=0.09)$ (Fig. $1 b)$. The average weighted UniFrac value within subjects of POI group was significantly higher than control group $(0.41 \pm 0.13$ versus $0.37 \pm 0.11, P<0.01)$ (Fig. $1 c$ ).

\section{Characterizing the gut microbiome in POI group}

Gut microbiome communities were dominated by phyla Firmicutes, Bacteroidetes, Actinobacteria and Proteobacteria in both POI and control groups (Fig. 2a). And Firmicutes was the predominant microbe, accounting for $65.35 \% \pm 18.71$ and $76.83 \% \pm 14.90 \%$ in POI and control group, respectively. The top 10 abundant genera in both two groups were Bacteroides, Bifidobacterium, Blautia, Clostridium, Coprococcus, Faecalibacterium, Megamonas, Prevotella, Roseburia and Ruminococcus (Fig. 2b). Compared to the control group, Bacteroides $(13.42 \% \pm$ $11.06 \%$ versus $8.45 \% \pm 10.14 \%)$, Bifidobacterium $(6.30 \% \pm$ $11.76 \%$ versus $5.0 \% \pm 8.84 \%$ ), Megamonas $(2.97 \% \pm 12.90 \%$ versus $1.37 \% \pm 4.61 \%)$, Prevotella $(4.09 \% \pm 9.90 \%$ versus $2.37 \% \pm 4.62 \%)$ increased, whereas Blautia $(9.60 \% \pm 7.37 \%$ versus $11.72 \% \pm 7.90 \%)$, Clostridium $(1.61 \% \pm 2.13 \%$ versus $4.31 \% \pm 7.99 \%)$, Coprococcus $(3.0 \% \pm 2.93 \%$ versus $3.91 \% \pm$ $4.12 \%)$, Faecalibacterium $(13.35 \% \pm 10.39 \%$ versus $21.65 \% \pm$ $14.80 \%)$, Roseburia $(3.63 \% \pm 4.72 \%$ versus $3.84 \% \pm 3.98 \%)$ and Ruminococcus $(3.73 \% \pm 3.46 \%$ versus $4.75 \% \pm 5.90 \%)$ decreased in POI group.

Through Wilcoxon Rank Sum tests (Fig. 2c), phylum Bacteroidetes $(21.10 \% \pm 14.01 \%$ versus $12.72 \% \pm 11.96 \%$, $P=0.04)$, genera Butyricimonas $(0.12 \% \pm 0.16 \%$ versus $0.04 \% \pm 0.08 \%, P=0.03)$, Dorea $(1.91 \% \pm 1.72 \%$ versus $1.02 \% \pm 0.83 \%, \quad P=0.049), \quad$ Lachnobacterium $(0.26 \% \pm$ $0.72 \%$ versus $0.0011 \% \pm 0.0047 \%, P=0.007)$ and Sutterella $(0.34 \% \pm 0.46 \%$ versus $0.08 \% \pm 0.11 \%, P=0.02)$ significantly increased in POI women, compared to control group. Whereas phylum Firmicutes $(P=0.03)$, genera Bulleidia $(0.0006 \% \pm 0.0033 \%$ versus $0.0094 \% \pm 0.021 \%$, $P=0.007)$ and Faecalibacterium $(P=0.04)$ significantly decreased. Moreover, Bacteroidetes/Firmicutes ratio $(0.41 \pm 0.44$ versus $0.20 \pm 0.24, P=0.03)$ in POI group was significantly higher than control group.

\section{Association between differential gut microbiota and clinical characteristics}

Pearson correlation analysis was performed after adjusting for BMI to evaluate the association between the differential microbes and serum hormones (Fig. 3). The results showed that E2 level was significantly negatively correlated with relative proportion of Bacteroidetes $(R=-0.41, P=0.002)$ and Bacteroidetes/Firmicutes ratio $(R=-0.45, P<0.01)$, while positively correlated with Firmicutes $(R=0.30, P=0.03)$ and Faecalibacterium $(R=0.28, P=0.047)$. FSH was significantly positively correlated with Bacteroidetes $(R=0.31, P=0.02)$, Bacteroidetes/Firmicutes ratio $(R=0.35, P=0.01)$, and negatively correlated with Firmicutes $(R=-0.28, P=0.04)$. LH 


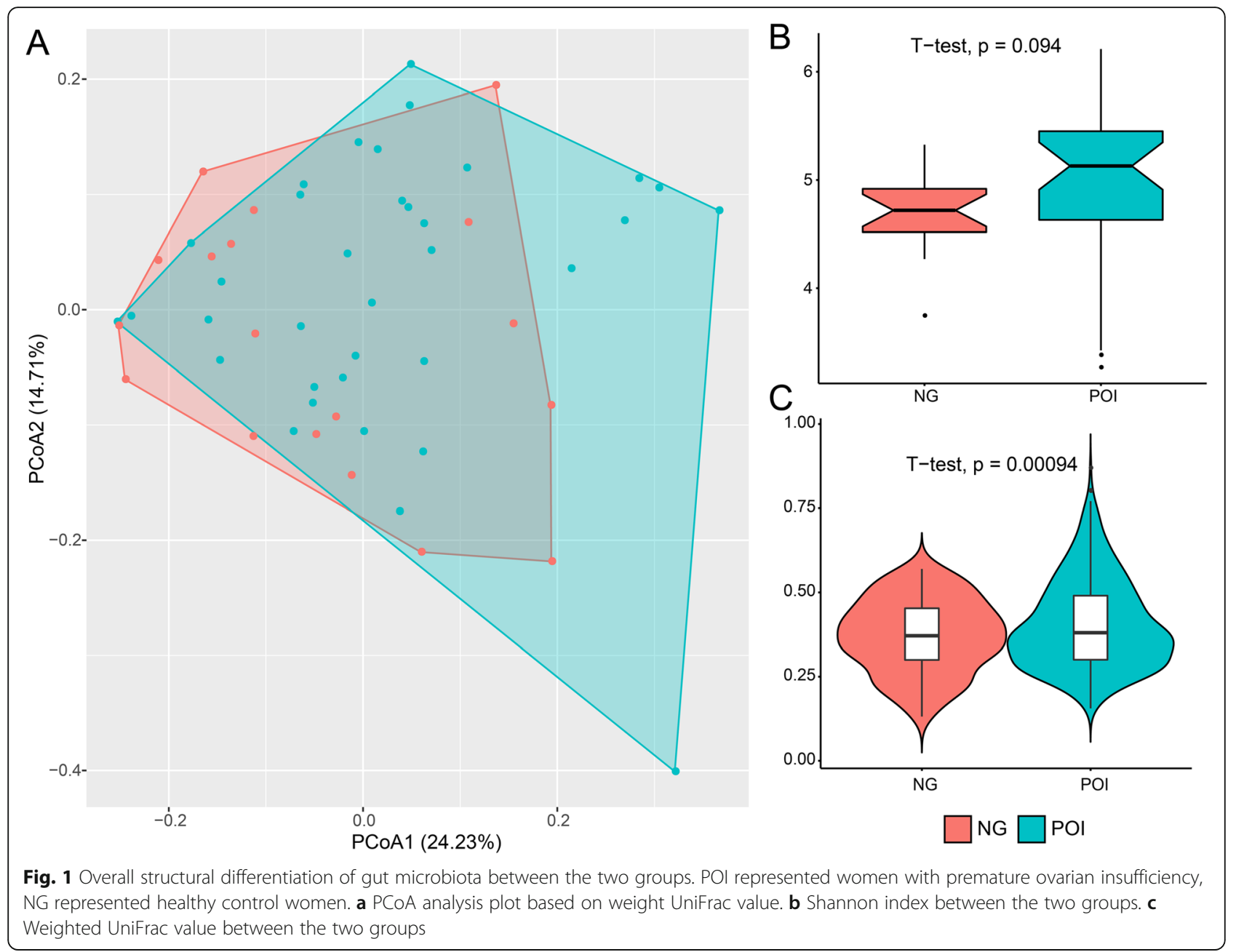

was significantly positively correlated with Bacteroidetes/Firmicutes ratio $(R=0.31, P=0.02)$. FSH/LH ratio was significantly positively correlated with the relative proportion of Bacteroidetes $(R=0.35, P=0.01)$, Bacteroidetes/Firmicutes ratio $(R=0.34, P=0.01)$, Dorea $(R=0.40, P<0.01)$, and negatively correlated with the relative proportion of Firmicutes $(R=-0.30, P=0.03)$ and Faecalibacterium $(R=-$ $0.34, P=0.01)$. AMH level was significantly correlated with the relative proportion of Bacteroidetes $(R=-0.28$, $P=0.04)$, Bacteroidetes/Firmicutes ratio $(R=-0.30, P=$ $0.03)$, Butyricimonas $(R=-0.31, P=0.02)$ and Faecalibacterium $(R=0.33, P=0.02)$.

\section{Discussion}

This study aimed to reveal the overall composition of gut microbiota in women with POI. As 15 to $30 \%$ of POI occurrences are considered to be familial [12]. To rule out genetic influences, all subjects recruited in this study were without blood relationship. Besides genetic factor, autoimmunity is an important etiology for POI, and gut microbiome is important for the development and maturation of the host immune system. In this study, the results showed that all recruited subjects composed primarily of phyla Actinobacteria, Bacteriodetes, Firmicutes and Proteobacteria. The sum relative abundance of Bacteriodetes and Firmicutes accounted for more than 90\%, which was consistent with previous studies [42, 1]. A balance between Bacteriodetes and Firmicutes is important to maintain intestinal homeostasis [1], while a significant higher Bacteriodetes/Firmicutes ratio was observed in POI subjects compared to control subjects. Furthermore, the abundant genera were Bacteroides, Bifidobacterium, Blautia, Clostridium, Coprococcus, Faecalibacterium, Megamonas, Prevotella, Roseburia and Ruminococcus. Most of them play important roles in the maintenance of host gastrointestinal homeostasis and health $[37,25]$. Notably, Bacteroides fragilis produce some peptides similar to type II collagen to induce crossreactive responses and to promote Th17 responses [45]. Similarly, some peptides produced by Bacteroides thetaiotaomicron and Roseburia intestinalis show similarity with human Ro60 and $\beta 2$-glycoprotein I, which can trigger lupus-like symptoms [36, 16]. Bifidobacterium 
A

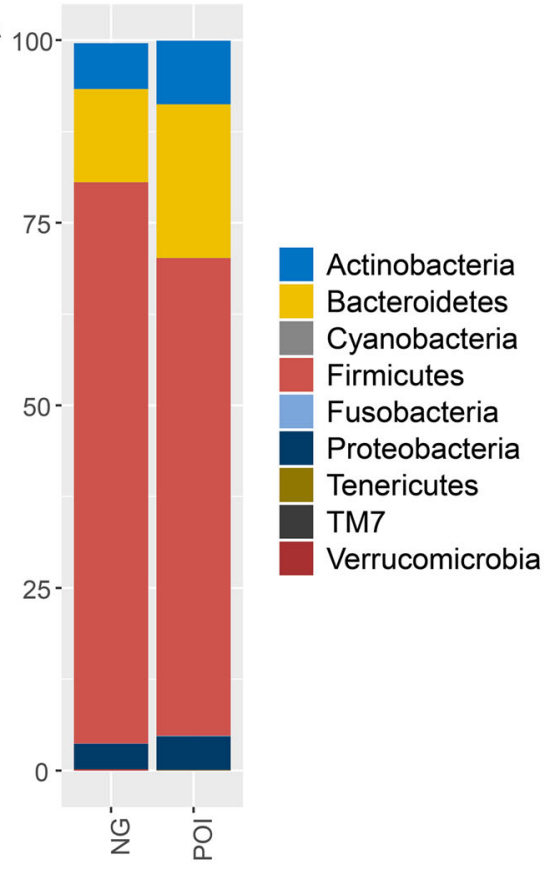

C

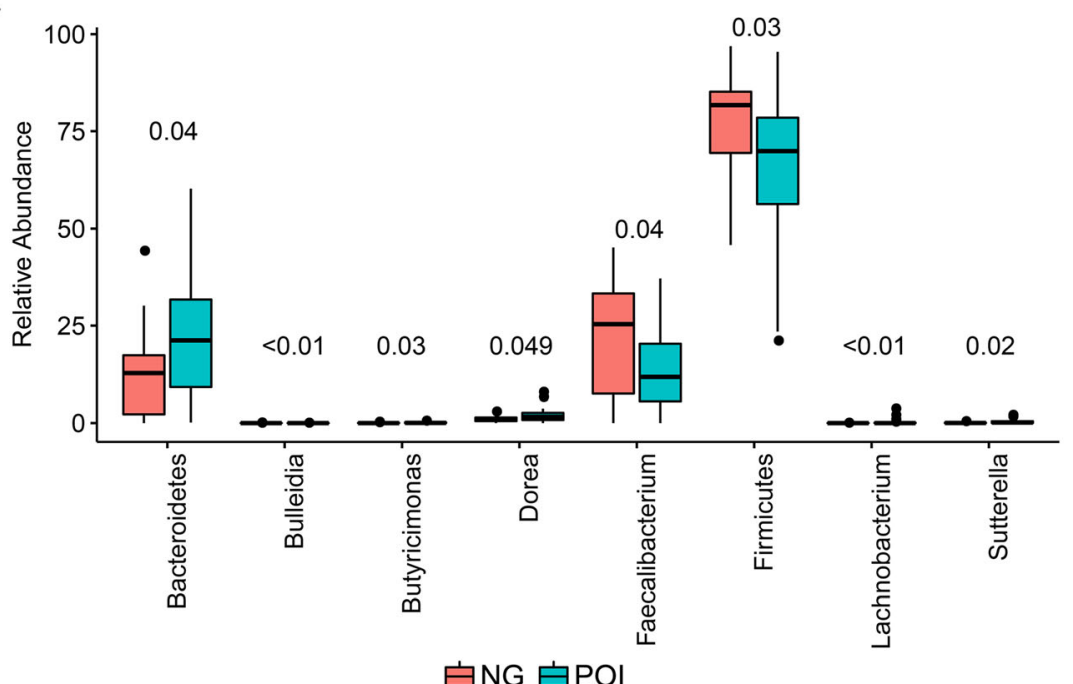

B

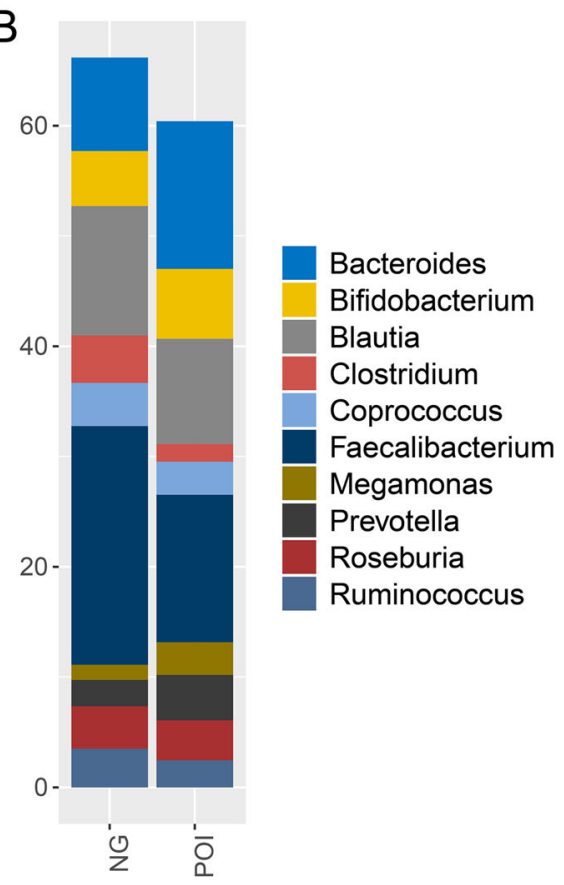

Fig. 2 Microbial community profiles of gut microbiota between POI and control group. POI represented women with premature ovarian insufficiency, NG represented healthy control women. a Relative abundances of the dominant phylum. $\mathbf{b}$ Relative abundances of the top 10 genera according to the relative abundance. c Significant different and important microbes between POI and control group

adolescentis can induce and promote Th17 responses in intestine [40]. Prevotella copri also can express antigens, and promote Th17 responses [27]. While the relative abundances of genera Bacteroides, Bifidobacterium and Prevotella all increased in POI group in this study. Moreover, genera Bifidobacterium, Blautia, Clostridium, Faecalibacterium, Roseburia and Ruminococcus can produce short chain fatty acids (SCFAs) in human gut, such as acetate, butyrate and succinate and so on [31, 35]. SCFAs not only have anti-inflammatory and immunomodulatory properties through modulating Treg/Th17 cell balance, but also can influence psychological function and cognitive processes [35, 7]. Butyrate was reported to alleviate antibody-induced arthritis in a natural killer $\mathrm{T}$ celldependent manner [23]. While the relative abundances of Blautia, Clostridium, Faecalibacterium, Roseburia and Ruminococcus presented decrease trends, especially Faecalibacterium decreased significantly in POI women in this study. All theses changes of gut microbiome in POI group might induce immunomodulating activity through certain 


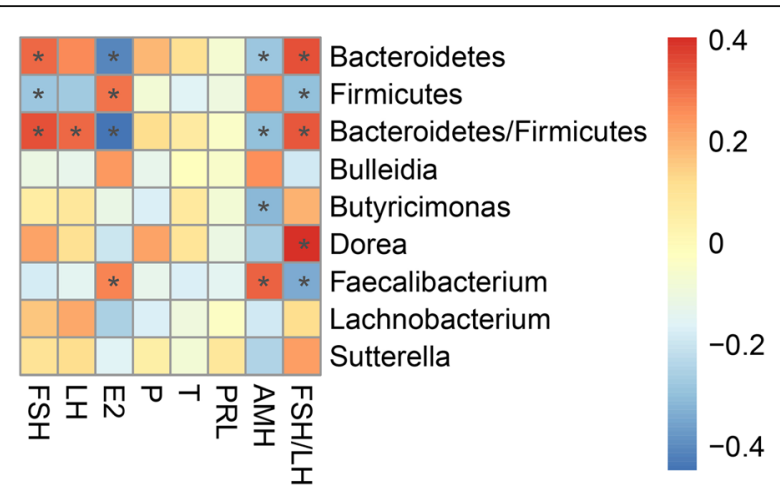

Fig. 3 Associations between gut microbiota and serum hormones. The star indicate the significant correlation at 5\% level

bacterial strains and their metabolites, which might be related to autoimmunity, then affects the development of POI.

Further, the gut microbiome is also found to be related to POI-associated autoimmune disorders [8]. In this study, there were significant decrease of Firmicutes, and significant increase of Bacteroidetes and Bacteroidetes/ Firmicutes ratio in POI women. It was consistent with the microbial community structure of type 1 diabetes and systemic lupus erythematosus patients, with a low proportion of Firmicutes, high proportion of Bacteroidetes and Bacteroidetes/Firmicutes ratio [10, 19]. Moreover, genera Dorea and Sutterella were more abundant in POI group. Increased proportion of Dorea is related to multiple multiple sclerosis, an autoimmune condition [6]. Sutterella is a mildly pro-inflammatory genus, and its elevated level is associated with cognitive function $[43,41]$. As a SCFAs producing member with antiinflammatory properties, Faecalibacterium decreased significantly in POI group in this study, its low proportion is also related to multiple sclerosis [5]. Butyrate producer Butyricimonas [9] increased significantly in POI group in this study. On the contrary, a low abundance of Butyricimonas was observed in multiple sclerosis fecal samples [13], that might be due to different species under Butyricimonas. Type 1 diabetes, systemic lupus erythematousus and multiple sclerosis, and cognitive dysfunction are all closely related to POI. Thus, the alterations of gut microbiome observed in this study might be related to the development of POI through affecting on the POI-associated diseases.

The gut microbiome has been shown to play an important part in impacting estrogen level through the secretion of $\beta$-glucuronidase, which could deconjugate estrogen and affect related physiological process $[32,1]$. In this study, significant lower level of E2 was observed in POI subjects, and it was significantly correlated with the proportion of Bacteroidetes, Firmicutes and Faecalibacterium by adjusting for BMI. And the FSH, LH and
$\mathrm{AMH}$ levels were also related to some microbes. It indicated that the altered gut microbiota of POI was associated with the sex hormones. Accumulating researches indicates that estrogen regulate glucose and lipid metabolism, bone formation and inflammatory response, its reduction can impair estrogen-dependent processes, triggering cardiovascular disease, osteoporosis and so on $[2,24]$. These symptoms are all related to POI. Yet due to limited data in this study, the mechanism under the relations between these microbes and sex hormones is not clear.

POI leads to several complications, including decrease in bone mineral density, autoimmune, thyroid disease risk and cognitive dysfunction. This study not only revealed the association between gut microbiota and autoimmunity, but also the relationship between gut microbiota and these complications. These indicated that the dysbiosis of gut microbiota was related to the development of POI discussed above. However, limited sample size, participants from the same hospital and the observation of association but not causality, large sample size and multi-center are needed in the further studies. Moreover, metagenome sequencing, measurements of metabolites produced by gut microbiota, animal experiments should also be considered to explore the potential causal mechanism.

\section{Conclusions}

In summary, POI may cause by autoimmune etiology, and the autoimmune process is affected by gut microbiome, so we may consider some relationship may exist between gut microbiome and POI.this study demonstrated an altered gut microbial pattern in women with POI against healthy controls, with an increase in Bacteroides, Bifidobacterium, Megamonas, Prevotella and a decrease in Blautia, Clostridium, Coprococcus, aecalibacterium, Roseburia and Ruminococcu. And we also found that these changes of microbes were closely related to serum hormones. This will help us to make a foundation for revealing the interaction between gut microbiota and POI certainly.

\section{Abbreviations}

BMI: Body mass index; FSH: Follicle-stimulating hormone; LH: Luteinizing hormone; E2: Estradiol; P: Progesterone; T: Testosterone; PRL: Prolactin; AMH: Anti-Mullerian hormone; GLU: Glucose

\section{Acknowledgements Not Applicable.}

\section{Authors' contributions}

J. L. Yao and Y. Ning conceived of the presented idea and planned the experiments. Y.Y. Zhuo and Y. Chen carried out the experiments. J.L.Yao and Y.L.Liu designed the computational framework and analyzed the data. J.M.Wu, J. L. Yao, Y. Ning and Y.Y. Zhuo wrote the manuscript. All authors discussed the results and contributed to the final manuscript. And all authors have read and approved the manuscript. 


\section{Funding}

This work was supported by Sanming Project of Medicine in Shenzhen (No. SZSM201612046), Guangdong Provincial Administration of Traditional Chinese Medicine (No. 20201294), and Internal research project of Shenzhen Maternity\&Child Healthcare Hospital (No. FYA2018006). The funders had no role in study design, data collection, analysis and interpretation, decision to publish, or preparation of the manuscript.

\section{Availability of data and materials}

The dataset supporting the conclusion of this article is available in the NCBI's Sequence Read Archive database (SRA BioProject ID PRJNA615330).

\section{Declarations}

\section{Ethics approval and consent to participate}

This study was approved by the Ethics Committee of Shenzhen

Maternity\&Child Healthcare Hospital (No. SFYLS [2020]012), Shenzhen, China. All patients provided written informed consent for their participation in the study. This study was registered at Chinese Clinical Trial Registry (registration No. ChiCTR2000029576)

\section{Consent for publication}

Not applicable.

\section{Competing interests}

The authors declare that they have no competing interests.

\section{Author details}

'Affiliated Shenzhen Maternity\&Child Healthcare Hospital, Southern Medica University, No. 3012, Fuqiang Road, Futian District, Shenzhen City 518000, Guangdong Province, China. ${ }^{2}$ Shenzhen Traditional Chinese Medicine Hospital, Shenzhen 518033, China.

Received: 5 August 2020 Accepted: 5 May 2021

Published online: 05 June 2021

\section{References}

1. Baker JM, Al-Nakkash L, Herbst-Kralovetz MM. Estrogen-gut microbiome axis: physiological and clinical implications. Maturitas. 2017;103:45-53. https://doi.org/10.1016/j.maturitas.2017.06.025

2. Baker L, Meldrum KK, Wang M, Sankula R, Vanam R, Raiesdana A, et al. The role of estrogen in cardiovascular disease. J Surg Res. 2003;115(2):325-44. https://doi.org/10.1016/S0022-4804(03)00215-4.

3. Bakhsh H, Dei M, Bucciantini S, Balzi D, Bruni V. Premature ovarian insufficiency in young girls: repercussions on uterine volume and bone mineral density. Gynecol Endocrinol. 2015;31(1):65-9. https://doi.org/10.31 09/09513590.2014.958987.

4. Bidet M, Bachelot A, Bissauge E, Golmard JL, Gricourt S, Dulon J, et al. Resumption of ovarian function and pregnancies in 358 patients with premature ovarian failure. J Clin Endocrinol Metab. 2011;96(12):3864-72. https://doi.org/10.1210/jc.2011-1038

5. Cantarel BL, Waubant E, Chehoud C, Kuczynski J, DeSantis TZ, Warrington J, et al. Gut microbiota in multiple sclerosis: possible influence of immunomodulators. J Investig Med. 2015;63(5):729-34. https://doi.org/10.1 097/JIM.0000000000000192

6. Chen J, Chia N, Kalari KR, Yao JZ, Novotna M, Paz Soldan MM, et al. Multiple sclerosis patients have a distinct gut microbiota compared to healthy controls. Sci Rep. 2016;6(1):28484. https://doi.org/10.1038/srep28484.

7. Dalile B, Van Oudenhove L, Vervliet B, Verbeke $K$. The role of short-chain fatty acids in microbiota-gut-brain communication. Nat Rev Gastroenterol Hepatol. 2019:16(8):461-78. https://doi.org/10.1038/s41575-019-0157-3.

8. De Luca F, Shoenfeld Y. The microbiome in autoimmune diseases. Clin Exp Immunol. 2019;195(1):74-85. https://doi.org/10.1111/cei.13158.

9. de Oliveira GLV, Leite AZ, Higuchi BS, Gonzaga MI, Mariano VS. Intestinal dysbiosis and probiotic applications in autoimmune diseases. Immunology. 2017:152(1):1-12. https://doi.org/10.1111/imm.12765.

10. Demirci M, Bahar Tokman H, Taner Z, Keskin FE, Çağatay P, Ozturk Bakar Y, et al. Bacteroidetes and Firmicutes levels in gut microbiota and effects of hosts TLR2/TLR4 gene expression levels in adult type 1 diabetes patients in Istanbul, Turkey. J Diabetes Complications. 2020;34(2):107449. https://doi. org/10.1016/j.jdiacomp.2019.107449.
11. Domniz N, Meirow D. Premature ovarian insufficiency and autoimmune diseases. Best Pract Res Clin Obstet Gynaecol. 2019;60:42-55. https://doi. org/10.1016/j.bpobgyn.2019.07.008.

12. Fenton A. Premature ovarian insufficiency: pathogenesis and management. J Life Health. 2015;6(4):147-53. https://doi.org/10.4103/0976-7800.172292.

13. Forbes JD, Van Domselaar G, Bernstein CN. The gut microbiota in immunemediated inflammatory diseases. Front Microbiol. 2016;7:1081. https://doi. org/10.3389/fmicb.2016.01081.

14. Galland L. The gut microbiome and the brain. J Med Food. 2014;17(12): 1261-72. https://doi.org/10.1089/jmf.2014.7000.

15. Goldmeier S, De Angelis K, Rabello Casali K, Vilodre C, Consolim-Colombo F, Belló Klein A, et al. Cardiovascular autonomic dysfunction in primary ovarian insufficiency: clinical and experimental evidence. Am J Transl Res. 2013;6(1): 91-101.

16. Greiling TM, Dehner $C$, Chen X, Hughes K, Iñiguez AJ, Boccitto $M$, et al. Commensal orthologs of the human autoantigen Ro60 as triggers of autoimmunity in lupus. Sci Transl Med. 2018;10:eaan2306. https://doi.org/1 0.1126/scitransImed.aan2306.

17. Guo T, Zheng Y, Li G, Zhao S, Ma J, Oin Y. Novel pathogenic mutations in minichromosome maintenance complex component 9 (MCM9) responsible for premature ovarian insufficiency. Fertil Steril. 2020;113(4):845-52. https:// doi.org/10.1016/j.fertnstert.2019.11.015.

18. Gupta A, Tiwari P. Premature ovarian insufficiency: a review. EMJ Reprod Health. 2019. https://doi.org/10.33590/emjreprohealth/19-00041.

19. He Z, Shao T, Li H, Xie Z, Wen C. Alterations of the gut microbiome in Chinese patients with systemic lupus erythematosus. Gut Pathog. 2016;8(1): 64. https://doi.org/10.1186/s13099-016-0146-9.

20. Ibáñez L, Rouleau M, Wakkach A, Blin-Wakkach C. Gut microbiome and bone. Joint Bone Spine. 2019;86(1):43-7. https://doi.org/10.1016/j.jbspin.201 8.02.008.

21. Kirshenbaum M, Orvieto R. Premature ovarian insufficiency (POI) and autoimmunity-an update appraisal. J Assist Reprod Genet. 2019:36(11):2207_ 15. https://doi.org/10.1007/s10815-019-01572-0.

22. Lach G, Schellekens H, Dinan TG, Cryan JF. Anxiety, depression, and the microbiome: a role for gut peptides. Neurotherapeutics. 2018;15(1):36-59. https://doi.org/10.1007/s13311-017-0585-0.

23. Lee S, Koh J, Chang Y, Kim HY, Chung DH. Invariant NKT cells functionally link microbiota-induced butyrate production and joint inflammation. J Immunol. 2019;203(12):3199-208. https://doi.org/10.4049/jimmunol.1801314.

24. Li JY, Chassaing B, Tyagi AM, Vaccaro C, Luo T, Adams J, et al. Sex steroid deficiency-associated bone loss is microbiota dependent and prevented by probiotics. J Clin Invest. 2016;126(6):2049-63. https://doi.org/10.1172/ JCl86062.

25. Lopetuso LR, Scaldaferri F, Petito V, Gasbarrini A. Commensal clostridia: leading players in the maintenance of gut homeostasis. Gut Pathog. 2013; 5(1):23. https://doi.org/10.1186/1757-4749-5-23.

26. Luisi S, Orlandini C, Regini C, Pizzo A, Vellucci F, Petraglia F. Premature ovarian insufficiency: from pathogenesis to clinical management. J Endocrinol Investig. 2015;38(6):597-603. https://doi.org/10.1007/s40618-0140231-1.

27. Maeda Y, Kurakawa T, Umemoto E, Motooka D, Ito Y, Gotoh K, et al. Dysbiosis contributes to arthritis development via activation of autoreactive T cells in the intestine. Arthritis Rheumatol. 2016;68(11):2646-61. https://doi. org/10.1002/art.39783.

28. Martin $C R$, Osadchiy $\vee$, Kalani A, Mayer EA. The brain-gut-microbiome axis. Cell Mol Gastroenterol Hepatol. 2018;6(2):133-48. https://doi.org/10.1016/j. jcmgh.2018.04.003.

29. Medina-Gomez C. Bone and the gut microbiome: a new dimension. J Lab Precis Med. 2018;3:96. https://doi.org/10.21037/jlpm.2018.11.03.

30. Mondul AM, Rodriguez C, Jacobs EJ, Calle EE. Age at natural menopause and cause-specific mortality. Am J Epidemiol. 2005;162(11):1089-97. https:// doi.org/10.1093/aje/kwi324

31. Parada Venegas D, De la Fuente MK, Landskron G, González MJ, Quera $\mathrm{R}$, Dijkstra $\mathrm{G}$, et al. Short chain fatty acids (SCFAs)-mediated gut epithelial and immune regulation and its relevance for inflammatory bowel diseases. Front Immunol. 2019:10:277. https://doi.org/10.3389/ fimmu.2019.00277.

32. Plottel CS, Blaser MJ. Microbiome and malignancy. Cell Host Microbe. 2011; 10(4):324-35. https://doi.org/10.1016/j.chom.2011.10.003.

33. Podfigurna-Stopa A, Czyzyk A, Grymowicz M, Smolarczyk R, Katulski K, Czajkowski K, et al. Premature ovarian insufficiency: the context of long-term 
effects. J Endocrinol Investig. 2016;39(9):983-90. https://doi.org/10.1007/s4 0618-016-0467-z.

34. Portman DJ, Gass MLS. Genitourinary syndrome of menopause: new terminology for vulvovaginal atrophy from the international society for the study of women's sexual health and the north American menopause society. Menopause. 2014;21(10):1063-8. https://doi.org/10.1097/GME. 0000000000000329

35. Ríos-Covián D, Ruas-Madiedo P, Margolles A, Gueimonde M, de los ReyesGavilán CG, Salazar N. Intestinal short chain fatty acids and their link with diet and human health. Front Microbiol. 2016;7:185. https://doi.org/10.3389/ fmicb.2016.00185.

36. Ruff WE, Dehner C, Kim WJ, Pagovich O, Aguiar CL, Yu AT, et al. Pathogenic autoreactive $T$ and $B$ cells cross-react with mimotopes expressed by a common human gut commensal to trigger autoimmunity. Cell Host Microbe. 2019;26(1):100-13. https://doi.org/10.1016/j.chom.2019.05.003.

37. Sarkar A, Pitchumoni CS. Identification of the microbiota in the aging process. In: The Microbiota in Gastrointestinal Pathophysiology: Elsevier; 2017. p. 37-56. https://doi.org/10.1016/B978-0-12-804024-9.00004-5.

38. Sassarini J, Lumsden MA, Critchley HOD. Sex hormone replacement in ovarian failure - new treatment concepts. Best Pract Res Clin Endocrinol Metab. 2015;29(1):105-14. https://doi.org/10.1016/j.beem.2014.09.010.

39. Siljander $\mathrm{H}$, Honkanen J, Knip M. Microbiome and type 1 diabetes EBioMedicine. 2019;46:512-21. https://doi.org/10.1016/j.ebiom.2019.06.031.

40. Tan TG, Sefik E, Geva-Zatorsky N, Kua L, Naskar D, Teng F, et al. Identifying species of symbiont bacteria from the human gut that, alone, can induce intestinal Th17 cells in mice. Proc Natl Acad Sci U S A. 2016;113(50):E814150. https://doi.org/10.1073/pnas.1617460113.

41. Ticinesi A, Tana C, Nouvenne A, Prati B, Lauretani F, Meschi T. Gut microbiota, cognitive frailty and dementia in older individuals: a systematic review. Clin Interv Aging. 2018;13:1497-511. https://doi.org/10.2147/CIA.S139163.

42. Turnbaugh PJ, Ley RE, Mahowald MA, Magrini V, Mardis ER, Gordon JI. An obesity-associated gut microbiome with increased capacity for energy harvest. Nature. 2006;444(7122):1027-31. https://doi.org/10.1038/na ture05414.

43. Wang L, Christophersen CT, Sorich MJ, Gerber JP, Angley MT, Conlon MA. Increased abundance of Sutterella spp. and Ruminococcus torques in feces of children with autism spectrum disorder. Mol Autism. 2013;4(1):42. https:// doi.org/10.1186/2040-2392-4-42.

44. Yorgun H, Tokgözoğlu L, Canpolat U, Gürses KM, Bozdağ G, Yapıcı Z, et al, The cardiovascular effects of premature ovarian failure. Int J Cardiol. 2013; 168(1):506-10. https://doi.org/10.1016/j.jicard.2012.09.197.

45. Zhang $X$, Chen B, Zhao L, Li H. The gut microbiota: emerging evidence in autoimmune diseases. Trends Mol Med. 2020;26(9):862-73. https://doi.org/1 0.1016/j.molmed.2020.04.001

46. Zheng P, Li Z, Zhou Z. Gut microbiome in type 1 diabetes: a comprehensive review. Diabetes Metab Res Rev. 2018;34(7):e3043. https:// doi.org/10.1002/dmrr.3043

\section{Publisher's Note}

Springer Nature remains neutral with regard to jurisdictional claims in published maps and institutional affiliations.

Ready to submit your research? Choose BMC and benefit from:

- fast, convenient online submission

- thorough peer review by experienced researchers in your field

- rapid publication on acceptance

- support for research data, including large and complex data types

- gold Open Access which fosters wider collaboration and increased citations

- maximum visibility for your research: over $100 \mathrm{M}$ website views per year

At BMC, research is always in progress.

Learn more biomedcentral.com/submissions 